\title{
Endoscopic monoportal removal of a choroid plexus papilloma in the posterior third ventricle in a child
}

\begin{abstract}
Albert A. Sufianov, MD, DSc, Saidi S. K. Gaibov, MD, and Rinat A. Sufianov
Federal State-Financed Institution "Federal Centre of Neurosurgery" of Ministry of Health of the Russian Federation, Tyumen, Russia

Currently, only a few reports describe the minimally invasive removal of choroid plexus papillomas (CPPs) and, to the best of the authors' knowledge, no reports detail the resection of such a papilloma through an endoscopic approach in infants. The authors here describe the endoscopic removal of a third ventricle CPP in a child. A 5-month-old male infant presented with progressive macrocephaly, vomiting, and convulsions. A lesion in the posterior third ventricle was detected on brain MRI. Because of the patient's very young age, neuroendoscopy was used as the least invasive technique. The tumor was completely resected through a monoportal neuroendoscopic approach. Histologically, the tumor was classified as a WHO Grade I CPP. After surgery, the patient's condition improved, with no complications during his recovery. Ten-month follow-up neuroimaging revealed no evidence of tumor recurrence or progressive hydrocephaly. In view of the successful neuroendoscopic excision of this posterior third ventricle CPP, the authors believe that this method seems promising in the treatment of young children with intraventricular lesions.
\end{abstract}

http://thejns.org/doi/abs/10.3171/2014.12.PEDS14306

KEY WORDS choroid plexus papilloma; third ventricle; neuroendoscopy; monoportal removal; technique

$\mathrm{C}$ HOROID plexus papilloma (CPP) is a benign neoplasm that stems from cells of the choroid plexus and has a neuroectodermal genesis. Its annual incidence is 0.3 case per 1 million persons,${ }^{10}$ and it constitutes $0.5 \%-0.6 \%$ of all intracranial tumors in all age groups. ${ }^{5,9}$ Few publications describe CPP removal in children via endoscopy either as an assisting tool or as an independent approach. Fully endoscopic removal of a third ventricle CPP has not been reported in the literature. In fact, third ventricle CPPs are rare, and their posterior location inside the third ventricle is even rarer. Here, we describe our experience in removing a posterior third ventricle CPP by adopting a fully endoscopic approach, and then we review the pertinent literature on the matter.

\section{Case Report}

History

A 5-month-old male infant was admitted to a local clinic when he developed generalized convulsive activity, anterior fontanelle bulging, and vomiting. Brain CT scanning revealed a mass in the third ventricle and internal hydrocephalus. His head circumference progressively increased. The boy regressed developmentally and was unable to roll over or hold his head up. He was transferred to the pediatric neurosurgical unit at the Federal Centre of Neurosurgery, Tyumen, several weeks later for further treatment. The period between the onset of symptoms and surgical intervention may have been used for diagnostic examination given the potential for slow progression of the disease. The delay may also be explained by geographical factors.

Upon admission to our institution, the boy remained unable to hold his head up or roll over. The patient had no complaints as regards the cardiovascular, respiratory, digestive, and urinary systems. His head circumference measured $46 \mathrm{~cm}$, and the anterior fontanelle was taut. Neurological assessment revealed convergent strabismus, a decrease in muscle strength to +4 in the upper and lower extremities bilaterally, a decrease in bilateral upper and lower extremity muscle tone, hyperactive reflexes in the upper and lower extremities, and a Babinski sign bilaterally. Fun-

ABBREVIATIONS CPP = choroid plexus papilloma; ETV = endoscopic ventriculocisternostomy.

SUBMITTED June 27, 2014. ACCEPTED December 31, 2014.

INCLUDE WHEN CITING Published online April 24, 2015; DOI: 10.3171/2014.12.PEDS14306.

DISCLOSURE The authors report no conflict of interest concerning the materials or methods used in this study or the findings specified in this paper. 
duscopic examination was negative for congestion in the ocular fundus. Magnetic resonance imaging of the brain revealed an irregularly shaped mass, $2.12 \times 1.61 \times 1.45 \mathrm{~cm}$ in size, inside the third ventricle, which had enlarged to $2.21 \mathrm{~cm}$. The mass had irregular, well-defined margins and was iso- to hypointense on T1-weighted imaging and mildly hyperintense on T2-weighted imaging. The mass occupied the posterior areas of the third ventricle, compressing the ventricular aqueduct and dislocating anterior aspects of the quadrigeminal plate into the cistern. Contrast injection revealed intense irregular enhancement of the mass. The lateral ventricles were enlarged asymmetrically, with the left larger than the right. Periventricular edema around the third and lateral ventricles was evident on T2-weighted and FLAIR imaging (Fig. 1).

\section{Operation}

The patient was selected to undergo monoportal endoscopic removal of the tumor, and the senior author performed an endoscopic ventriculocisternostomy (ETV) via the floor of the third ventricle. The patient was placed supine with his head immobilized inside a Doro clamp (PMI). We used endoscopic instruments for cranial surgeries: EndoWorld Neuro 12-E Lotta and Hopkins II $0^{\circ}$ and $30^{\circ}$ rigid endoscopes (both Karl Storz). Surgical entry into the anterior horn of the right lateral ventricle was performed parasagittally from the right frontal area via a bur hole. A neuroendoscope was then advanced inside the cranium. Tumor located in the third ventricle was visualized via the foramen of Monro (10 $\mathrm{mm}$ in diameter). Because MRI indicated that the tumor was protruding into the ventricular aqueduct and occluding it, we initially considered only a partial tumor resection. Given the occlusive nature of the hydrocephalus and in an effort to control its progression and avoid the need for a CSF diversion surgery in the future, we performed an ETV using a standard technique. Upon examination of the foramen of Monro and the third ventricle, we discovered that tumor tissue was intimately connected to the thalamostriate vein and the choroid plexus. After coagulation, the tumor tissue was sent for histological testing.
Working bimanually and using biopsy forceps, endoscopic scissors, and a bipolar coagulation electrode, we dissected the tumor and isolated the vascular pedicle, which we cauterized and resected. Then the neoplasm was completely removed via the foramen of Monro. Upon examination, the third ventricle was free of tumor tissue, and the ventricular aqueduct was well visualized with no evidence of occlusion (Fig. 2). Estimated blood loss was $15 \mathrm{ml}$. Analysis of the CSF collected intraoperatively revealed cytosis (1 neutrophil present), protein $11 \mathrm{mg} / \mathrm{dl}$, chloride $115 \mathrm{mmol} / \mathrm{L}$, and glucose $48.6 \mathrm{mg} / \mathrm{dl}$.

\section{Postoperative Course}

The patient's postoperative recovery was satisfactory. Brain MRI 3 days after the operation confirmed total resection of the tumor and a third ventricle size of $1.67 \mathrm{~cm}$. There was still evidence of moderate periventricular edema around the anterior horns of the lateral ventricles (Fig. 3 ). At the time of discharge on postoperative Day 9, the patient was more active and without strabismus. Muscle strength was still +4 in the upper and lower extremities bilaterally with decreased muscle tone in all extremities. He had normal reflexes in the upper and lower extremities, with age-appropriate normal plantar reflex responses bilaterally. His appetite improved, and his head circumference had decreased by $1.5 \mathrm{~cm}$. At the 10 -month follow-up visit, there was no progression of the hydrocephalus and no evidence of tumor recurrence.

\section{Pathological Findings}

Histological examination revealed papillary structures with a large number of dilated blood vessels with hyperemia. The surface was lined with a single layer of cuboidal epithelium, which was morphologically consistent with a WHO Grade I choroid papilloma (Fig. 4).

\section{Discussion}

Meng et al. reported on the endoscopic removal of a cystic CPP of the third ventricle in an 18-year-old patient, with no evidence of recurrence during 2 years of follow-up observation. ${ }^{16}$ Reddy et al. described a combined approach
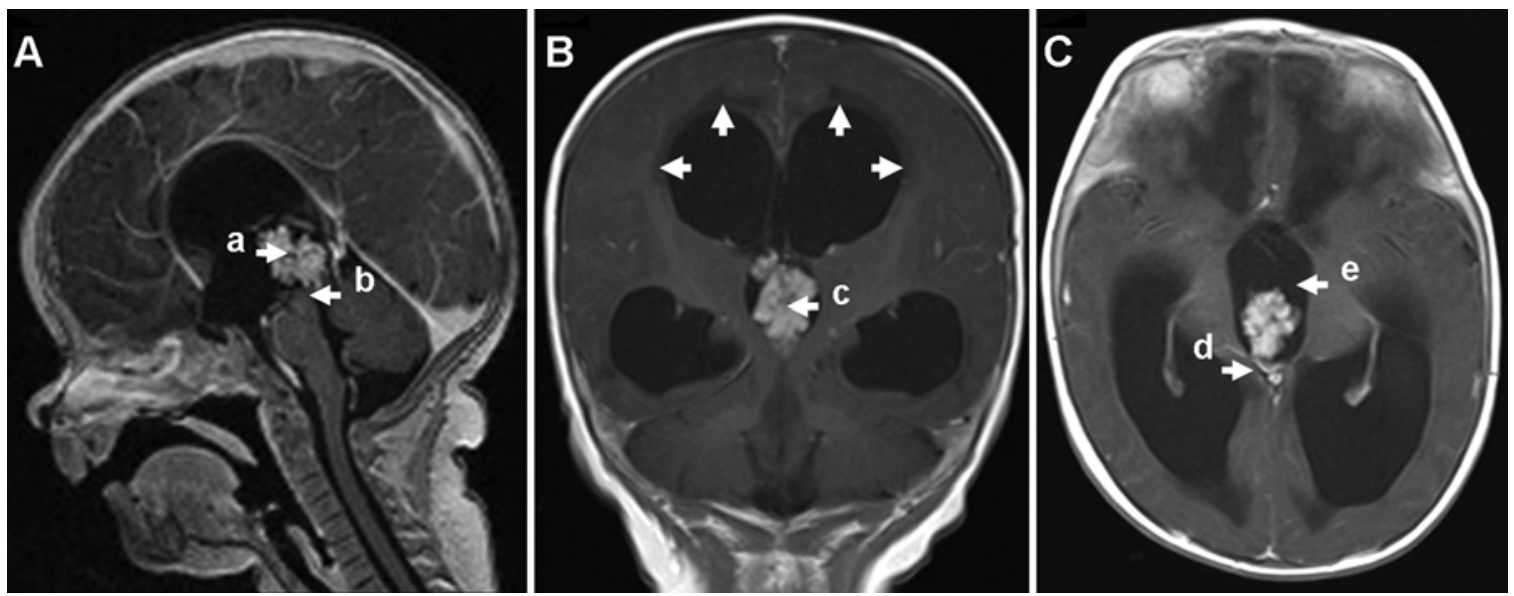

FIG. 1. Brain MRI with intravenous contrast before surgery. A: Sagittal image showing tumor (a) located in the posterior aspect of the third ventricle. Tumor penetration (b) into the cerebral aqueduct resulted in a blockage. B: Coronal image showing a cauliflower-like tumor nodule (c) in the third ventricle in the setting of hydrocephalus. Arrows indicate periventricular edema. C: Axial image showing the vascular pedicle of the tumor (d). Note the tumor in relation to the size of the third ventricle (e). 

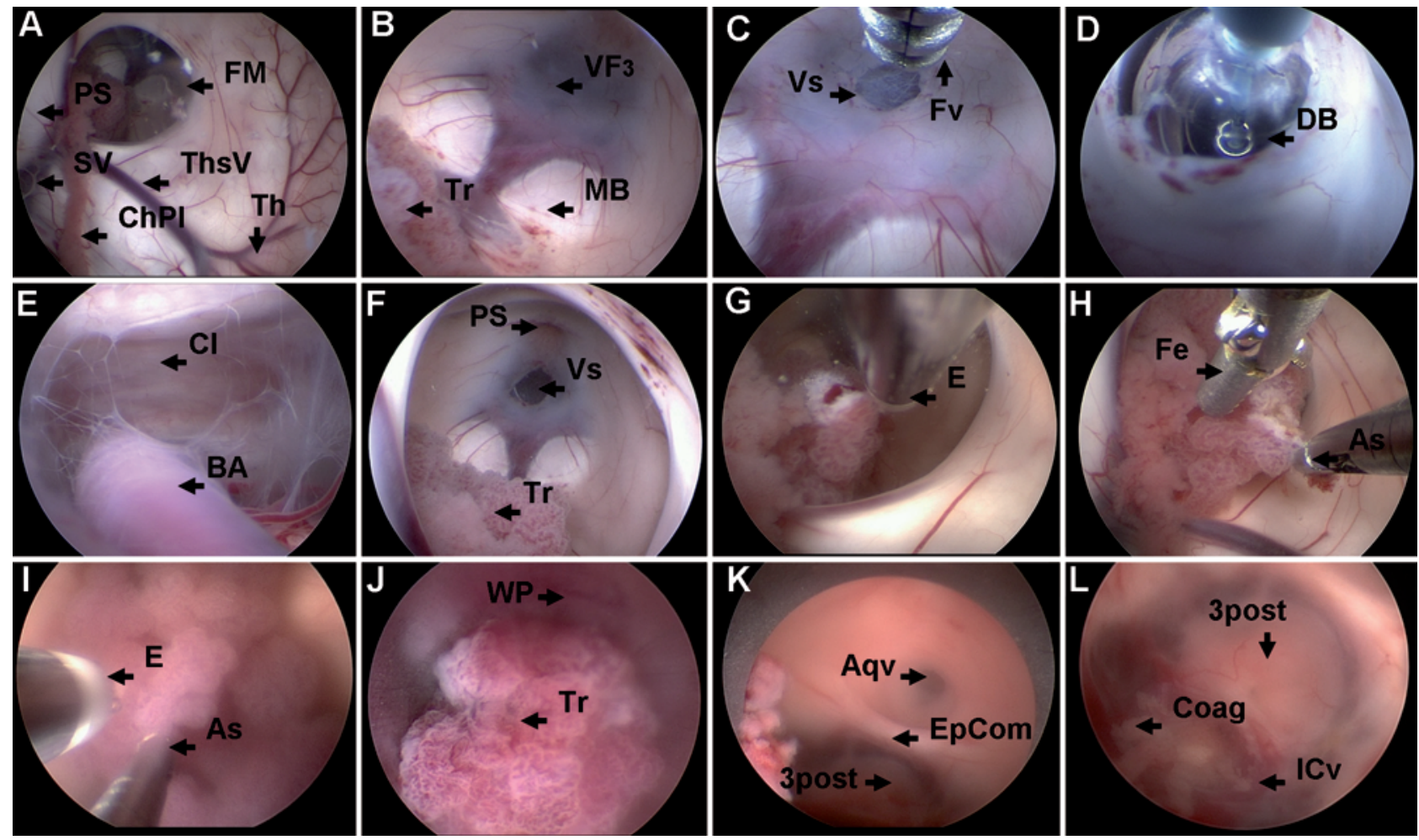

FIG. 2. Main steps in the endoscopic monoportal removal of a CPP from the posterior third ventricle. A: The main anatomical intraventricular landmarks. ChPI = choroid plexus; FM = foramen of Monro; PS = pellucid septum; SV = septal vein; Th = thalamus; ThsV = thalamostriate vein. B: Relation between the main anatomical landmarks of the third ventricle and the tumor. MB $=$ mammillary bodies; $\mathrm{Tr}=$ tumor; VF3 = ventricular floor of the third ventricle. C: Perforation of the ventricular floor of the third ventricle (first stage of ETV). Fv = ventriculostomy forceps; $V s=$ formed ventriculostomy. D: Dilation of ventriculostomy (second stage of ETV). DB = double balloon ventriculostomy catheter. E: Interpeduncular cistern revision (third stage of ETV). BA = basilar artery; $\mathrm{Cl}=$ clivus. $\mathrm{F}$ : View of third ventricle after ETV. PS = pituitary stalk; $\mathrm{Tr}=$ tumor; $\mathrm{Vs}=$ ventriculostomy. G: Beginning of tumor removal and partial coagulation. $\mathrm{E}=$ bipolar electrode. $\mathrm{H}$ : Bimanual endoscopic microsurgical dissection of the tumor. As = aspirator; $\mathrm{Fe}=$ endoscopic biopsy forceps. I: Removal of tumor after its morcellation with the help of a coagulator and a surgical aspirator. As = aspirator; E = bipolar electrode. J: Removed tumor in the lumen of the working port channel (WP). Tr $=$ tumor mass. K: Endoscopic view of posterior third ventricle after removal of the tumor. Aqv = cerebral aqueduct; EpCom = epithalamic commissure; 3 post $=$ posterior of the third ventricle (ventricular roof of third ventricle). L: Tumor bed after removal of the tumor. Coag = area of coagulated vascular pedicle of tumor; ICv = internal cerebral vein.

to a third ventricle CPP in a 6-month-old patient with secondary hydrocephalus, initially using endoscopy for biopsy and mobilization of the tumor from the third ventricle to the right lateral ventricle and then openly removing the lesion via a transcortical approach. ${ }^{22}$

In half of all pediatric or adult cases, tumor is located in the lateral ventricle of the brain. In $40 \%$ of cases, the tumor may occur in the fourth ventricle. Choroid plexus papillomas in the third ventricle compose only $5 \%-8 \%$ of all cases. ${ }^{1,14}$ The remaining 5\% of cases may be found in various brain regions, including the cerebellopontine angle, suprasellar region, frontal lobe, epithalamic commissure, pineal region, and cerebellum. ${ }^{12}$ According to many authors, CPPs localized in the third ventricle constitute a very rare subset of all CPPs. ${ }^{3,11,19,21,23}$ Fortuna et al. reviewed 4000 brain tumor surgeries in the Institute of Neurosurgery at the Catholic University in Rome, where only 1 case of CPP occurred inside the third ventricle. ${ }^{6}$

Eighty percent of third ventricular papillomas are located in the anterior region of the third ventricle, ${ }^{6,8,11,24}$ and a posterior localization of CPPs in the third ventricle is very rare. ${ }^{17}$
According to Galassi et al., 14\% of choroid plexus tumors occur during the 1st year of life. ${ }^{7}$ However, Nakano et al. described a CPP in the posterior aspect of the third ventricle in a 42-year-old woman, and Tomasello et al. treated a 49-year-old man with the same CPP localization. ${ }^{17,24}$

Choroid plexus papillomas located in the third ventricle have their own characteristics. As the third ventricle is small and complex in its anatomy, it presents certain difficulties for surgical tumor removal. ${ }^{4}$ Moreover, given the anatomical features of the third ventricle, the clinical presentation of tumors localized there occurs earlier than for tumors inside the lateral ventricles. ${ }^{25}$ Among children with such lesions, one of the most common features is the development of hypertensive hydrocephalus. Among older patients, symptoms include vision impairment, gait disturbances, and other general symptoms associated with increased intracranial pressure and direct tumor pressure. ${ }^{2,18}$ Schijman et al. analyzed 10 CPP cases in the third ventricle in children, and all of them had hydrocephalus. ${ }^{23}$

Brain imaging shows CPPs to be round formations with tuberous surfaces. On both MRI and CT scans, CPPs 

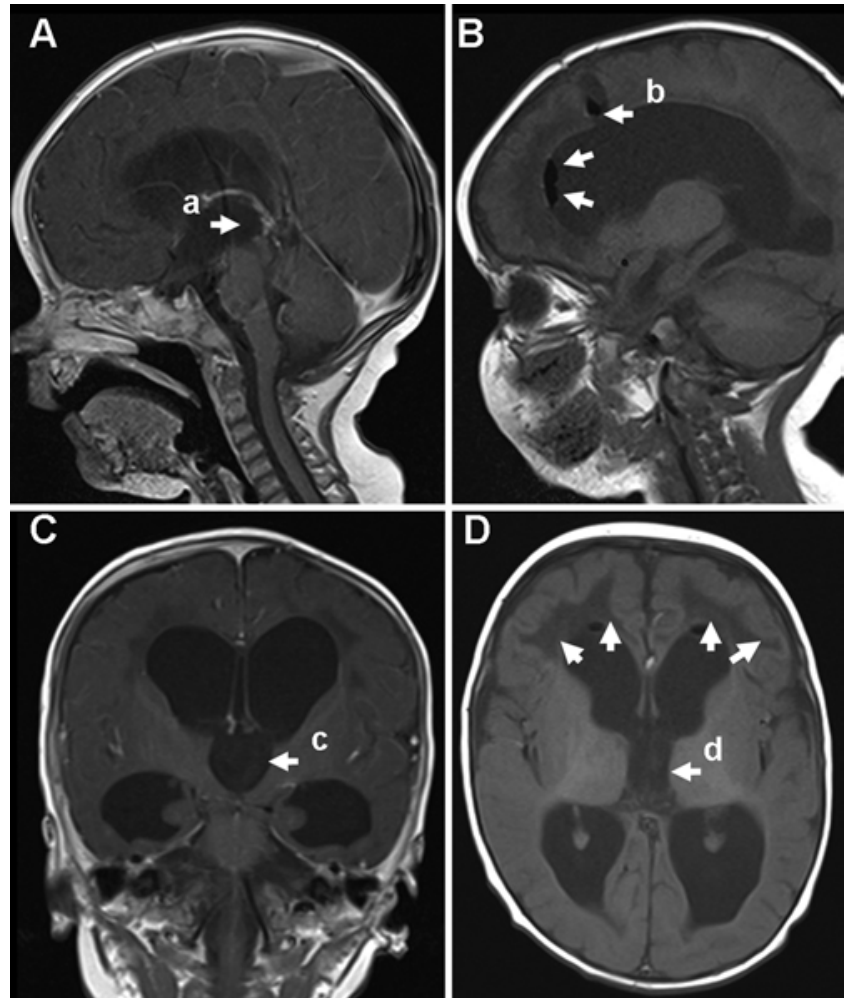

FIG. 3. Brain MRI with intravenous contrast after surgery. A: Sagittal section showing the tumor completely removed. Location of tumor prior to its removal (a). B: Sagittal section endoscopic hole (b) after transcortical monoportal access. Arrows indicate air in the anterior parts of the lateral ventricles. C: Coronal section of third ventricle (c), free of tumor. D: Axial section showing a decreased size of the third ventricle after tumor removal and regression of the internal hydrocephalus. Arrows indicate residual signs of the periventricular edema. Lumen of the third ventricle $(d)$.

present as shapes with well-defined, large lobular margins. Computed tomography scans reveal a hyperdense structure. On MRI, T1-weighted sequences usually show a tumor that is isointense with the brainstem and T2-weighted sequences show a moderately hyperintense structure that can blend with the CSF in the image. Because the choroid papilloma is always highly vascularized, the injection of contrast leads to a notable increase in visualization. Tumor calcifications occur in $14 \%-25 \%$ of cases but are rarely seen in children..$^{12,13,15}$ Blood supply to CPPs of the posterior areas of the third ventricles comes from the medial and lateral posterior choroidal arteries and the collicular arteries. Venous drainage is achieved via the internal cerebral veins, basal vein of Rosenthal, choroid veins (from the system of the vein of Galen), and anteromedial occipital veins. ${ }^{26}$

Because of the benign nature of the neoplasm and its low recurrence rates, patient survival rates after $\mathrm{CPP}$ resection are high $(90 \%, 81 \%$, and $77 \%$ for 1-, 5-, and 10-year survival rates, respectively). ${ }^{27}$ The most common postoperative complications are subdural hygroma and the need for CSF drainage. In a report by Pencalet et al., ventriculoperitoneal shunting was required in $50 \%$ of patients. ${ }^{20}$

Surprisingly, few reports in the literature discuss en-

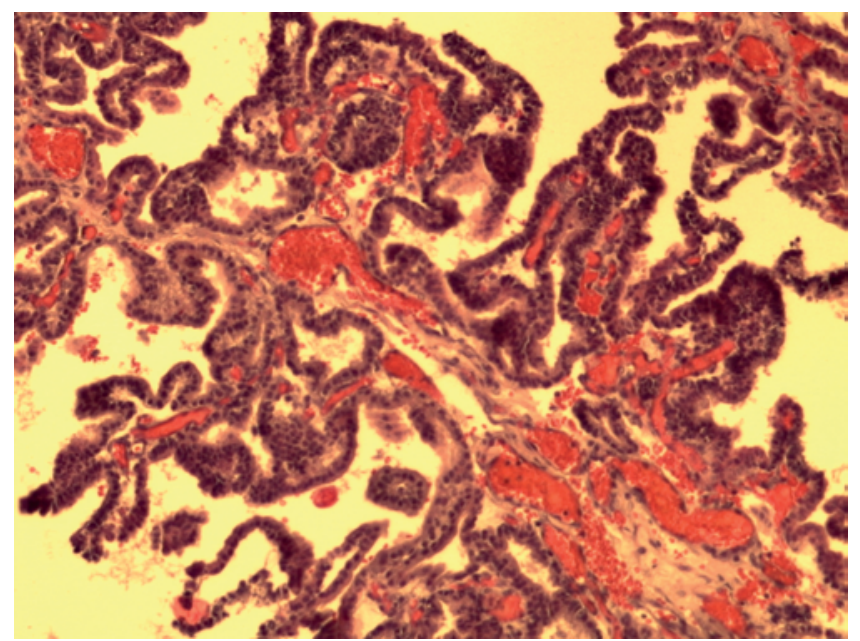

FIG. 4. Photomicrograph of a WHO Grade I CPP. H \& E, original magnification $\times 10$.

doscopic CPP removal. We believe that current endoscopy equipment is technically adequate for handling such surgeries. Our experience with successful endoscopic removal of CPPs - not only from the third but also from one of the lateral ventricles-leads us to certain conclusions about the use of the neuroendoscopic approach. First, preoperative planning requires determining whether there is enough space around the neoplasm to allow safe surgical manipulations. To establish that, one must compare the relative volumes of the tumor and the ventricle as well as the size and type of endoscope (rigid, semirigid, flexible, channeled, and so forth). One must also assess the accessibility of the vascular pedicle by the endoscopic instruments and verify that it can be safely manipulated without causing damage to it or other neurovascular structures. Additionally, one must decide on the number of necessary access ports, for example, when the vascular pedicle is difficult to reach.

Moreover, the following factors are important to consider before the operation: 1) whether a bimanual technique must be used (that is, whether 2 instruments must be manipulated at the same time during a multiportal approach with multiple instruments); 2) whether it will be possible to change the trajectory and the angle of attack using different portals of access, if necessary; and 3) whether one can reliably clip and/or cauterize the vascular pedicle during the operation. Note that when dealing with a large tumor, we morcellate it and then remove it together with the port through the endoscopic hole of the working port.

\section{Conclusions}

Choroid plexus papillomas of the third ventricle are very rare compared with those in other locations. For this reason, even a single successful surgical treatment using a minimally invasive technique in a young pediatric patient is of great practical interest. Additionally, the clinical case described here demonstrates that a minimally invasive technique can be successfully used for complete monoportal endoscopic resection of a CPP in the posterior third ventricular regions. 


\section{References}

1. Aguzzi A, Brandner S, Paulus W: Choroid plexus tumors, in Kleihues P, Cavenee W (eds): Pathology and Genetics of Tumours of the Nervous System. Lyon: IARC Press, 2000, pp 84-86

2. Boyd MC, Steinbok P: Choroid plexus tumors: problems in diagnosis and management. J Neurosurg 66:800-805, 1987

3. Carson BS, Weingart JD, Guarnieri M, Fisher PG: Third ventricular choroid plexus papilloma with psychosis. Case report. J Neurosurg 87:103-105, 1997

4. Do HM, Marx WF, Khanam H, Jensen ME: Choroid plexus papilloma of the third ventricle: angiography, preoperative embolization, and histology. Neuroradiology 43:503-506, 2001

5. Felix I, Phudhichareonrat S, Halliday WC, Becker LE: Choroid plexus tumors in children: immunohistochemical and scanning-electron-microscopic features. Pediatr Neurosci 13:263-269, 1987

6. Fortuna A, Celli P, Ferrante L, Turano C: A review of papillomas of the third ventricle. One case report. J Neurosurg Sci 23:61-76, 1979

7. Galassi E, Godano U, Cavallo M, Donati R, Nasi MT: Intracranial tumors during the 1st year of life. Childs Nerv Syst 5:288-298, 1989

8. Gradin WC, Taylon C, Fruin AH: Choroid plexus papilloma of the third ventricle: case report and review of the literature. Neurosurgery 12:217-220, 1983

9. Gupta N: Choroid plexus tumors in children. Neurosurg Clin N Am 14:621-631, 2003

10. Jänisch W, Staneczek W: [Primary tumors of the choroid plexus. Frequency, localization and age.] Zentralbl Allg Pathol 135:235-240, 1989 (Ger)

11. Jooma R, Grant DN: Third ventricle choroid plexus papillomas. Childs Brain 10:242-250, 1983

12. Koeller KK, Sandberg GD: From the archives of the AFIP. Cerebral intraventricular neoplasms: radiologic-pathologic correlation. Radiographics 22:1473-1505, 2002

13. Kornienko VN, Pronin IN: Supratentorial tumors, in Diagnostic Neuroradiology, Vol 2. Moscow: Burdenko Neurosurgical Institute, 2009, pp 333-488

14. Levy ML, Goldfarb A, Hyder DJ, Gonzales-Gomez I, Nelson M, Gilles FH, et al: Choroid plexus tumors in children: significance of stromal invasion. Neurosurgery 48:303-309, 2001

15. McEvoy AW, Harding BN, Phipps KP, Ellison DW, Elsmore AJ, Thompson D, et al: Management of choroid plexus tumours in children: 20 years experience at a single neurosurgical centre. Pediatr Neurosurg 32:192-199, 2000

16. Meng H, Feng H, Zhang L, Wang J: Endoscopic removal of a cystic choroid plexus papilloma of the third ventricle: a case report and review of the literature. Clin Neurol Neurosurg 113:582-585, 2011

17. Nakano I, Kondo A, Iwasaki K: Choroid plexus papilloma in the posterior third ventricle: case report. Neurosurgery 40:1279-1282, 1997

18. Pascual-Castroviejo I, Villarejo F, Perez-Higueras A, Morales C, Pascual-Pascual SI: Childhood choroid plexus neoplasms. A study of 14 cases less than 2 years old. Eur J Pediatr 140:51-56, 1983

19. Pawar SJ, Sharma RR, Mahapatra AK, Lad SD, Musa MM: Choroid plexus papilloma of the posterior third ventricle during infancy \& childhood: report of two cases with management morbidities. Neurol India 51:379-382, 2003

20. Pencalet P, Sainte-Rose C, Lellouch-Tubiana A, Kalifa C, Brunelle F, Sgouros S, et al: Papillomas and carcinomas of the choroid plexus in children. J Neurosurg 88:521-528, 1998

21. Razzaq AA, Chishti KN: Giant choroid plexus papilloma of the third ventricle. J Pak Med Assoc 53:573-575, 2003

22. Reddy D, Gunnarsson T, Scheinemann K, Provias JP, Singh SK: Combined staged endoscopic and microsurgical approach of a third ventricular choroid plexus papilloma in an infant. Minim Invasive Neurosurg 54:264-267, 2011

23. Schijman E, Monges J, Raimondi AJ, Tomita T: Choroid plexus papillomas of the III ventricle in childhood. Their diagnosis and surgical management. Childs Nerv Syst 6:331-334, 1990

24. Tomasello F, Albanese V, Bernini FP, Picozzi P: Choroid plexus papilloma of the third ventricle. Surg Neurol 16:6971,1981

25. Tomita T, McLone DG, Flannery AM: Choroid plexus papillomas of neonates, infants and children. Pediatr Neurosci 14:23-30, 1988

26. Verbova LN, Shaversky AV: [Choroid plexus papilloma of the posterior third ventricle.] Ukrainian Neurosurgical Journal 4:26-29, 2004 (Ukranian)

27. Wolff JE, Sajedi M, Brant R, Coppes MJ, Egeler RM: Choroid plexus tumours. Br J Cancer 87:1086-1091, 2002

\section{Author Contributions}

Conception and design: Gaibov, RA Sufianov. Acquisition of data: RA Sufianov. Analysis and interpretation of data: Gaibov. Drafting the article: Gaibov, RA Sufianov. Critically revising the article: all authors. Reviewed submitted version of manuscript: AA Sufianov. Approved the final version of the manuscript on behalf of all authors: AA Sufianov. Administrative/technical/ material support: AA Sufianov. Study supervision: AA Sufianov.

\section{Correspondence}

Albert A. Sufianov, Federal State-Financed Institution "Federal Centre of Neurosurgery" of Ministry of Health of the Russian Federation (Tyumen), Ul. 4 km. Chervishevskogo trakta, Tyumen 625032, Russia. email: sufianov@gmail.com. 